\title{
Application of boundary detection information in breast tomosynthesis reconstruction
}

\author{
Yiheng Zhang, ${ }^{\text {a) }}$ Heang-Ping Chan, Berkman Sahiner, Yi-Ta Wu, Chuan Zhou, Jun Ge, \\ Jun Wei, and Lubomir M. Hadjiiski \\ Department of Radiology, University of Michigan, Ann Arbor, Michigan 48109-0904
}

(Received 6 February 2007; revised 27 June 2007; accepted for publication 28 June 2007; published 22 August 2007)

\begin{abstract}
Digital tomosynthesis mammography (DTM) is one of the most promising techniques that can potentially improve early detection of breast cancers. DTM can provide three-dimensional (3D) structural information by reconstructing the whole imaged volume from a sequence of projectionview (PV) mammograms that are acquired at a small number of projection angles over a limited angular range. Our previous study showed that simultaneous algebraic reconstruction technique (SART) can produce satisfactory tomosynthesized image quality compared to maximum likelihoodtype algorithms. To improve the efficiency of DTM reconstruction and address the problem of boundary artifacts, we have developed methods to incorporate both two-dimensional (2D) and 3D breast boundary information within the SART reconstruction algorithm in this study. A second generation GE prototype tomosynthesis mammography system with a stationary digital detector was used for PV image acquisition from 21 angles in $3^{\circ}$ increments over a $\pm 30^{\circ}$ angular range. The 2D breast boundary curves on all PV images were obtained by automated segmentation and were used to restrict the SART reconstruction to be performed only within the breast volume. The computation time of SART reconstruction was reduced by $76.3 \%$ and $69.9 \%$ for cranio-caudal and mediolateral oblique views, respectively, for the chosen example. In addition, a 3D conical trimming method was developed in which the 2D breast boundary curves from all PVs were back projected to generate the 3D breast surface. This 3D surface was then used to eliminate the multiple breast shadows outside the breast volume due to reconstruction by setting these voxels to a constant background value. Our study demonstrates that, by using the 2D and 3D breast boundary information, all breast boundary and most detector boundary artifacts can be effectively removed on all tomosynthesized slices. (C) 2007 American Association of Physicists in Medicine.
\end{abstract}

[DOI: $10.1118 / 1.2761968]$

Key words: digital tomosynthesis mammography (DTM), image reconstruction, simultaneous algebraic reconstruction technique (SART), breast boundary detection, boundary artifact reduction

\section{INTRODUCTION}

Currently mammography is the only proven method used for breast cancer screening. However, a major limitation of mammography is that the projection images, recorded on a screen-film system (conventional mammography) or by a digital detector (digital mammography), only contain twodimensional (2D) projection of three-dimensional (3D) anatomical structures. As a result, the sensitivity of breast cancer detection is adversely affected by the camouflaging of cancerous lesions by dense breast tissues.

Digital tomosynthesis mammography (DTM) is one of the most promising techniques that can potentially improve early detection of breast cancers. ${ }^{1-5}$ DTM can provide 3D structural information by reconstructing the whole imaged volume from a sequence of projection-view (PV) mammograms that are acquired at a small number of projection angles over a limited angular range. The total radiation dose is set to be comparable to that used in regular screening mammography. It has been demonstrated ${ }^{6-8}$ that DTM can reduce the camouflaging effect of the overlapping fibroglandular breast tissue, thus improving the conspicuity of subtle lesions. Several prototype DTM systems have been developed based on digital mammography systems ${ }^{5,9,10}$ and preliminary pilot clinical trials are being conducted to evaluate the utility of DTM. ${ }^{11-13}$

The reconstruction of the $3 \mathrm{D}$ volume of the compressed breast from a sequence of 2D projection images in DTM represents a limited-angle cone-beam tomographic problem. Current reconstruction methods for such a problem include

- Back-projection-type algorithms, including the shiftand-add method used in the original tomosynthesis ${ }^{1,14}$ and multiple projection method; ${ }^{15}$

- Fourier-transform based algorithms, including the filtered back-projection (FBP) method ${ }^{9,16,17}$ and other transfer function methods, such as inversion filtering ${ }^{10,18,19}$ and matrix inversion tomosynthesis (MITS); $;^{20-22}$

- Algebraic reconstruction techniques (ARTs), including the original $\mathrm{ART}^{16,23}$ and simultaneous $\mathrm{ART}$ $(\mathrm{SART}) ;{ }^{24,25}$

- Statistical reconstruction algorithms, including maximum likelihood (ML) method with convex algorithm. ${ }^{26,27}$ 
Due to the limited angular range employed in DTM acquisition, only incomplete projection information of the imaged breast is available which results in intra- and inter- slice artifacts. The inter-slice artifacts come from the superposition of out-of-plane features on the in-focus plane which cannot be removed by DTM reconstruction alone. Many artifact reduction algorithms have been investigated, especially those to address artifacts of high attenuation objects, including deblurring techniques, ${ }^{28-31}$ order statistics operator, ${ }^{32,33}$ and voting strategies, ${ }^{34}$ and these methods have been incorporated into various reconstruction methods.

A review of tomosynthesis reconstruction techniques can be found in Dobbins and Godfrey. ${ }^{20}$ In our previous work, ${ }^{25}$ we have investigated three representative methods for DTM reconstruction based on breast phantom study and a second generation GE prototype DTM system: the back-projection method, the SART and the maximum likelihood method with the convex algorithm (ML-Convex). Our comparative study suggested that both SART and ML-Convex methods can achieve good-quality reconstruction and the SART method can provide comparable tomosynthesized image quality to those of ML-Convex method but with fewer number of iterations.

Two important aspects should be considered in breast tomosynthesis reconstruction. First, the demand on high spatial resolution results in a very high dimensionality of the inverse problem and prohibitive computational burden. It is therefore important to investigate methods to avoid unnecessary computation. Some parallel computation method ${ }^{27}$ and hardware acceleration techniques ${ }^{35}$ have been recently developed to improve the speed of DTM reconstruction. However, these methods are usually dedicated to specific reconstruction algorithms and the improvement depends on the algorithm under consideration. Since these dedicated software or hardware cannot be easily developed for every reconstruction technique, it is of practical importance to design methods to improve the computational efficiency, especially at the stage when the reconstruction algorithm is still being developed. Second, the DTM reconstruction is a severely underdetermined and ill-posed inverse problem due to the limited number of projections and the limited angular range which only provides incomplete projection information of the imaged breast. As a result, tomosynthesis reconstruction generally contains strong artifacts. These artifacts are represented as repeating ghosts along the $\mathrm{x}$-ray path directions on all tomosynthesized slices with reduced intensity. These artifacts also appear at the breast boundary in which the pixel intensity changes abruptly from breast tissue to air on PV images. The breast boundary artifacts, although easily distinguishable from breast anatomical features, could be distracting for radiologists' reading. Furthermore, these artifacts could negatively affect computerized processing of DTM images, e.g., computer-aided detection of mass lesions by using DTM reconstruction images. ${ }^{25,36}$

In this work, we developed a 3D conical trimming method based on back projection of the breast boundaries detected on the 2D PV images to generate the 3D breast surface and extract the breast volume. We investigated the application of

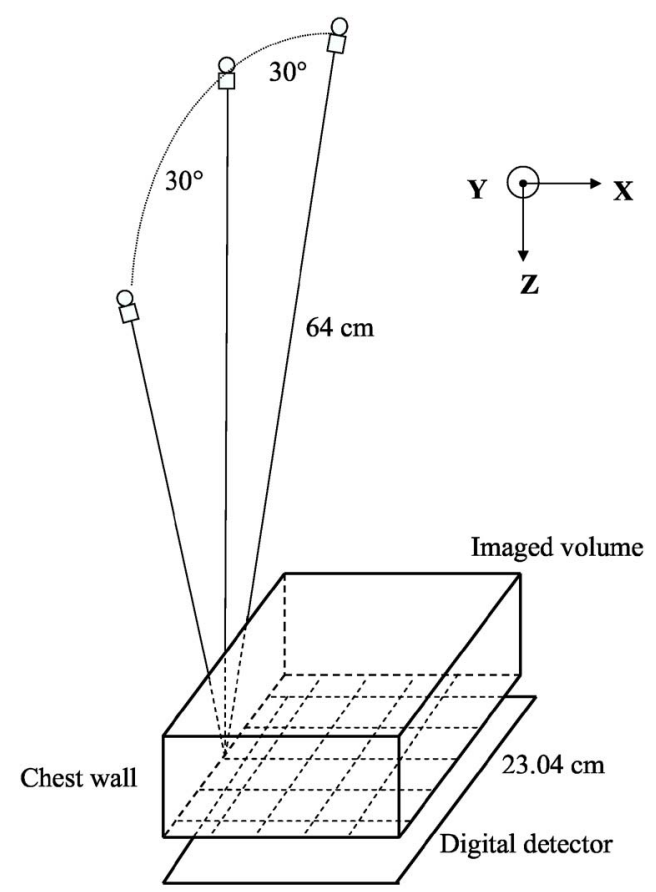

$19.2 \mathrm{~cm}$

FIG. 1. Geometry of the second generation GE prototype digital tomosynthesis mammography system used in this study.

the $2 \mathrm{D}$ and $3 \mathrm{D}$ breast boundary information to DTM reconstruction in an effort to reduce computational burden and to suppress boundary artifacts. With 2D breast boundary information on PV images, the reconstruction algorithm will only consider the $\mathrm{x}$-rays that intercept the breast volume. This will eliminate unnecessary computation outside the breast while keeping all useful information for reconstruction. Using patient DTM images, we demonstrate that the 2D and 3D information can be used to effectively remove two boundary artifacts: breast boundary artifacts and detector boundary artifacts, from the reconstructed DTM slices.

\section{MATERIALS AND METHODS}

\section{II.A. Breast tomosynthesis system}

The imaging geometry of the second generation GE prototype digital tomosynthesis system for breast imaging research is shown in Fig. 1. The DTM system has a CsI phosphor/a:Si active matrix flat panel digital detector with dimensions of $19.20 \mathrm{~cm} \times 23.04 \mathrm{~cm}$ and a pixel size of $0.1 \mathrm{~mm} \times 0.1 \mathrm{~mm}$. The digital detector is stationary during image acquisition. The DTM system acquires PV images at 21 different angles over a $\pm 30^{\circ}$ angular range by automatically rotating the $x$-ray tube in $3^{\circ}$ increments. The distance from the $\mathrm{x}$-ray focal spot to the center of rotation is $64 \mathrm{~cm}$ and the plane along which the $\mathrm{x}$-ray source rotates is perpendicular to the detector surface at the chest wall edge. The focal-spot-to-detector distance is $66 \mathrm{~cm}$. The image acquisition process takes less than $8 \mathrm{~s}$.

In our reconstruction algorithm, we define the "imaged volume" as a rectangular box having the same area as the 


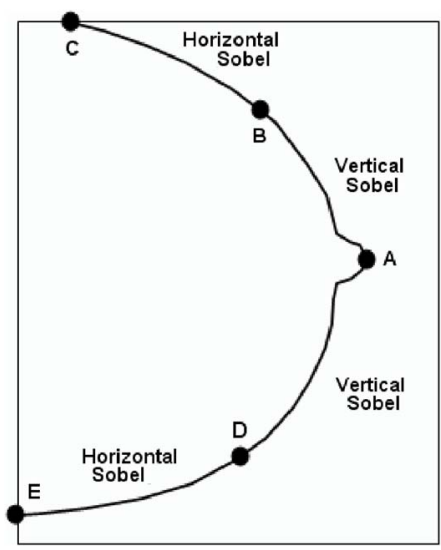

FIG. 2. The use of vertical and horizontal Sobel filtering in the breast boundary tracking procedure.

detector, and the same thickness as that of the compressed breast measured by the DTM system, as shown in Fig. 1. This imaged volume is subdivided into an array of voxels, of which the $X$ and $Y$ dimensions are chosen to be the same as the pixel size of the detector $(0.1 \mathrm{~mm} \times 0.1 \mathrm{~mm})$ while the $Z$ dimension (the slice thickness) is chosen to be $1 \mathrm{~mm}$ in this study.

As described in our previous paper, ${ }^{25}$ for the forward projection model, we have developed an algorithm to calculate the path length of a primary $\mathrm{x}$-ray intersecting each voxel within the imaged volume lattice, similar to Siddon's algorithm. ${ }^{37}$ Logarithmic transformation is applied to the raw pixel intensities of the detected image before reconstruction in the SART method. We assume a monoenergetic x-ray source and ignore the effects of scattering and beam hardening in this study, similar to the approach by Wu et al. ${ }^{2,38}$

\section{II.B. Detection of 2D breast boundary and generation of 3D breast surface}

The $2 \mathrm{D}$ breast boundary on a PV image is segmented by a breast boundary detection program developed in our laboratory $^{39,40}$ and adapted to suit DTM application. There are two main steps in the algorithm. In the first step, the initial breast boundary is obtained by applying Otsu's thresholding method ${ }^{41}$ to the histogram of the input image. The initial boundary is only a rough estimate of the boundary location so that gray level thresholding is sufficient. In the second step, a breast boundary tracking procedure is performed, using the initial boundary as a guide, by extracting gradient information from horizontal and vertical Sobel filtering. The application of the Sobel filters is illustrated in Fig. 2. The right-most pixel of the initial boundary is first determined, as indicated by point A in Fig. 2. Although this point coincides with the nipple location in this figure, it can be any point along the boundary. The tracking procedure starts from point A and moves upward and downward, respectively, to determine the final breast boundary. The vertical Sobel filter is used to enhance the edges in the ranges between $\mathrm{A}$ and $\mathrm{B}$ and between $\mathrm{A}$ and $\mathrm{D}$, and the horizontal Sobel filter is used in the ranges between $\mathrm{B}$ and $\mathrm{C}$ and be-

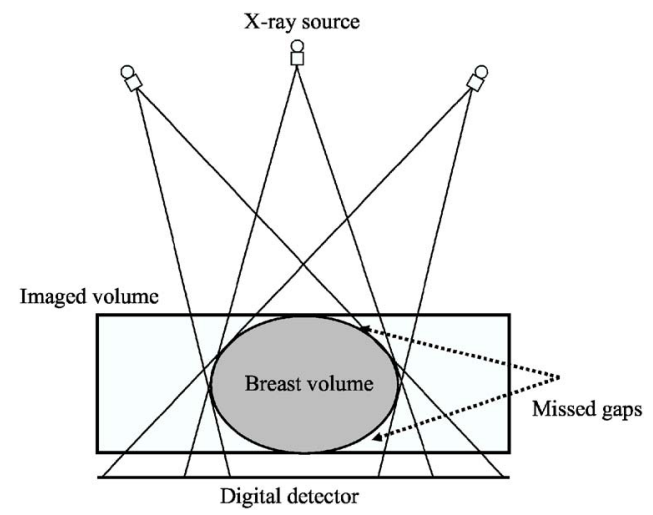

FIG. 3. Estimation of the 3D breast surface from 2D breast boundary curves by $3 \mathrm{D}$ conical trimming of the volume outside the breast. Dashed arrows indicate the potential missed gaps for which the breast surface cannot be recovered.

tween D and E. The selection of either the vertical or horizontal Sobel filter is determined based on the slope of the tangent to the current boundary tracking position. From our experience, this relatively simple boundary detection method is reliable for the DTM PV images we have processed so far. However, the focus of this work is not on the development of image segmentation methods. Our breast surface reconstruction algorithm described below does not depend on the boundary detection method so that any satisfactory breast boundary curves for DTM PV images obtained with our method or other segmentation techniques can be used.

With the detected 2D breast boundary curves on all PV images, we can generate a 3D breast surface within the imaged volume to enclose the compressed breast while excluding the exterior air space. In principle, there exists a unique convex hull inside the 3D imaged volume for the DTM system of which the projections at different angles precisely correspond to the breast shadow on each PV image. To recover this convex hull, we implement a " $3 \mathrm{D}$ conical trimming" process. This process is schematically depicted in Fig. 3 where only three x-ray source positions are drawn for illustration. Specifically, for a given PV image, the breast boundary curve is back projected into the imaged volume and all "air volume" outside the generalized cone surface formed by the x-ray source and the breast boundary is trimmed off. This process will repeat for all PV images. As the $\mathrm{x}$-ray source moves from one to the next position, the back-projected breast surface will trim off more air-volume in addition to the preceding ones. After all PV breast boundary curves have been used exactly once, the surface of the remaining volume becomes the desired convex hull and its projection with respect to each $\mathrm{x}$-ray source position corresponds to exactly the breast shadow. The convex hull combined with the top and bottom surfaces of the breast delineated by the compression paddle and the breast support plate define an enclosed breast volume.

Note that for any $x$-ray source position, some parts of the imaged volume will not be included in the cone-beam ray path, and at larger projection angles, some parts of the projected image will be cut off due to the limited detector size. 


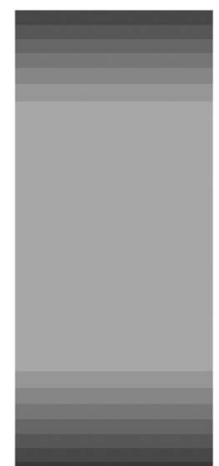

(a)

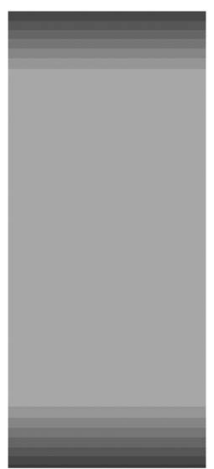

(b)

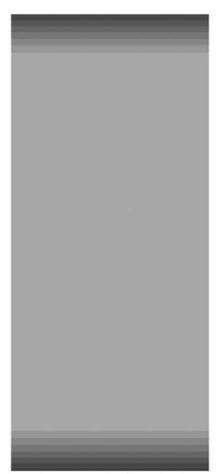

(c)
FIG. 4. PV-count maps of three selected depths: (a) $5.0 \mathrm{~cm}$, (b) $2.5 \mathrm{~cm}$, and (c) $1.0 \mathrm{~cm}$ above the breast support plate. The darker pixel intensity represents a fewer number of effective PVs. For all three slices, the top-most area contains only eight PVs and the bottom-most area contains only seven PVs. This number gradually increases to 21 from both ends to the middle region.

This problem is accentuated in a DTM system with a stationary detector. For different $\mathrm{x}$-ray source positions, the unexposed parts of the imaged volume change. The overall effect is that some middle part of the imaged volume is completely inside the x-ray path at all times, i.e., exposed by all $21 \mathrm{PVs}$; and for other parts, it is exposed by only a subset of the 21 PVs. Figure 4 shows examples of the PV-count maps, in which the intensity of a given pixel is proportional to the number of PVs contributing radiation to this pixel. The PVcount maps of three tomosynthesized slices at depths, 5.0, 2.5 , and $1.0 \mathrm{~cm}$, above the breast support plate, are shown. It can be seen that all PVs contribute to the middle region of each tomosynthesized slice, and this region grows larger as the distance from the support plate decreases. The number of steps on each side is determined by the imaging geometry of the DTM system, and is equal to the number of PVs of which the x-ray field boundary intersects the imaged volume on that side. Furthermore, for any tomosynthesized slice, the closer the region is to the imaged volume boundary, the fewer the number of PVs will expose the region, as apparent from the DTM geometry. In this specific example that we acquired with our GE prototype DTM system, only eight PVs can expose the uppermost while seven for the lowest boundary area on all tomosynthesized slices. Therefore 13 and 14 steps, respectively, can be seen on the upper and lower sides of the PV-count map. The unequal PV-count numbers on the upper and lower boundary areas may be attributed to the slightly asymmetric angular positions of the $\mathrm{x}$-ray source relative to the central location.

According to the 3D conical trimming process, for the fully exposed parts of the imaged volume (in terms of PVs), the $3 \mathrm{D}$ breast surface tangential to the $\mathrm{x}$ rays can be generated by the intersection of all back-projected 2D breast boundary curves. Thus, a voxel can be considered to be inside the breast volume if and only if its projection is within the breast boundary in all PVs. In contrast, in any partially exposed parts of the imaged volume, a voxel is considered to be inside the breast volume as long as its projection is within the breast boundary of any PV. This process ensures that any potential breast volume which may be contained only in some of the PV images due to the detector cutoff will be included. All voxels in the air volume above and below the breast volume will be excluded by the breast boundaries defined by the compression paddle and the breast support plate.

\section{II.C. Simultaneous algebraic reconstruction technique (SART) and boundary artifacts}

SART method ${ }^{24,42}$ is an iterative reconstruction technique. It was proposed as a refinement of the original ART. ${ }^{16,43,44}$ In SART method, an update of the 3D distribution of the attenuation properties of the imaged volume is performed based on each PV. The calculated current projection will be compared to the actual detection data and the difference will be back projected and added to the imaged volume distribution. The updating is performed after all rays in one PV have been processed, resulting in a "block-iterative" strategy. In contrast, ART method updates the distribution based on a rayby-ray manner. The number of updates in one complete iteration of SART is equal to the number of PVs.

Let the whole image volume be subdivided into $J$ voxels, and the linear attenuation coefficient for the $j$ th voxel be denoted by $x_{j}, 1 \leq j \leq J$; the digital area detector contains $I$ elements, and the $i$ th ray, $1 \leq i \leq I$, is defined as a line segment starting from the point $\mathrm{x}$-ray source location to the center of the $i$ th detector element. The number of rays is equal to the number of detector elements assuming one ray is traced for each element. The path length of the $i$ th ray going through the $j$ th voxel in the $n$th $\mathrm{x}$-ray tube location (projection view) is denoted by $a_{i j, n}$, resulting in a matrix-vector form of the projection model as

$$
\mathbf{A}_{n} \mathbf{x}=\mathbf{y}_{n},
$$

where $\mathbf{A}_{n}$ is the projection matrix for the $n$th PV with $a_{i j, n}$ as its $(i, j)$ th element; $\mathbf{y}_{n}$ is the corresponding vector of the projection data, which can be derived from the pixel values of the detected image, $1 \leq n \leq N$, where $N$ is the total number of PVs. The $i$ th projection value, $y_{i, n}$, is proportional to the logarithmic transform of the ratio of the incident intensity $\left(I_{o, n}\right)$ and the transmitted intensity $\left(I_{i, n}\right)$ of the $i$ th ray

$$
y_{i, n}=k \ln \frac{I_{o, n}}{I_{i, n}} .
$$

We stack the projection model in Eq. (1) for all PVs together as

$$
\left[\begin{array}{c}
\mathbf{A}_{1} \\
\vdots \\
\mathbf{A}_{N}
\end{array}\right] \mathbf{x}=\left[\begin{array}{c}
\mathbf{y}_{1} \\
\vdots \\
\mathbf{y}_{N}
\end{array}\right] \rightarrow \mathbf{A x}=\mathbf{y} .
$$

With the linear system model in Eq. (3), the SART method can be formulated in a matrix-vector form as

$$
\mathbf{x}^{n}=\mathbf{x}^{n-1}+\lambda \cdot \mathbf{M}_{n} \mathbf{A}_{n}^{T} \mathbf{W}_{n}\left(\mathbf{y}_{n}-\mathbf{A}_{n} \mathbf{x}^{n-1}\right),
$$

where $\mathbf{M}_{n} \in R^{J \times J}$ and $\mathbf{W}_{n} \in R^{I \times I}$ are diagonal matrices containing diagonal elements as $\left[\mathbf{M}_{n}\right]_{j j}=\left(\sum_{i=1}^{I} a_{i j, n}\right)^{-1}$ and $\left[\mathbf{W}_{n}\right]_{i i}=\left(\sum_{j=1}^{J} a_{i j, n}\right)^{-1}$, respectively. One can see that $\mathbf{M}_{n}$ and 
$\mathbf{W}_{n}$ contain inverse "column sum" and "row sum" of the projection matrix $\mathbf{A}_{n}$ that correspond to the total path length of all $\mathrm{x}$-rays through one imaged voxel and the total path length of one x-ray through the entire imaged volume, respectively. The updating strategy of SART is easy to understand based on Eq. (4) as follows: the calculated projection $\mathbf{A}_{n} \mathbf{x}^{n-1}$ is compared to the current PV data $\mathbf{y}_{n}$; the difference $\left(\mathbf{y}_{n}-\mathbf{A}_{n} \mathbf{x}^{n-1}\right)$ is normalized by the total path length of each $\mathrm{x}$-ray $\mathbf{W}_{n}$, yielding the estimated weighted average difference in the linear attenuation coefficient of the voxels along the ray. This average difference is back projected to the imaged volume by $\mathbf{A}_{n}^{T}$, and then for any given voxel, the differences are further normalized by the total path lengths of all rays traveling through this voxel by $\mathbf{M}_{n}$. The voxel is updated as the sum of the current estimation $\mathbf{x}^{n-1}$ and the weighted average difference scaled by a relaxation parameter $\lambda$. The updating operation starts from an initial distribution $\mathbf{x}^{0}$ and is repeated for all PV images using the corresponding projection matrices. One complete iteration is defined as the process that all PV images have been sequentially used exactly once. In our previous work, ${ }^{25}$ we experimentally demonstrated that SART method can provide satisfactory tomosynthesized image quality with one complete iteration using all PV images and $\lambda=0.5$.

In this work, we focus on two types of boundary artifacts: one from the breast boundary and the other from the detector boundary. Breast boundary artifacts are caused by the large difference in the pixel intensities in the two areas inside and outside the 2D breast boundary, corresponding to projection of overlapping breast tissues and air volume, respectively. Due to the uncertainty in the true 3D location of any feature within the imaged volume based on a limited number of PV images over a limited acquisition angular range, when back projected by DTM reconstruction methods, these boundaries in the PVs will result in repeating ghost artifacts of similar breast shape but reduced intensity on all tomosynthesized image slices.

Detector boundary artifacts are caused by the DTM system geometry in which a stationary finite-size detector acquires PV images of the breast from moving $\mathrm{x}$-ray source positions, as discussed in Sec. II B. In every PV position, a part of the imaged volume is not exposed by the cone-beam ray path and recorded by the detector, as shown in Fig. 5(a). The unexposed part of the imaged volume varies as the x-ray source moves. When we reconstruct the imaged volume using the SART method, ${ }^{25}$ the voxel values will be updated iteratively by processing each individual PV image. For a given PV, only the part of the imaged volume within the current field of view will be updated. Since the voxels within the PV image boundary (i.e., the detector boundary) will be changed whereas the neighboring voxels outside will maintain their previous values, it will cause a discontinuity in the voxel values across the image boundary. Similar situation occurs when the breast image exceeds the detector area and the real breast boundary is outside the detector boundary, as schematically shown in Fig. 5(b). This situation occurs most frequently on the PV images associated with large projection

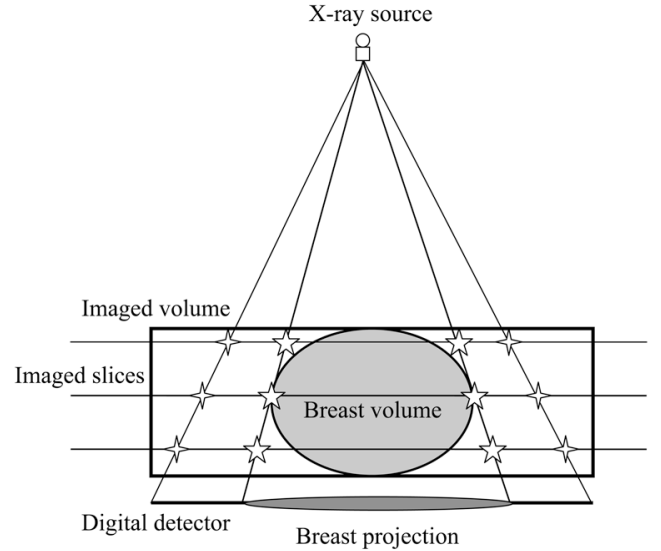

(a)

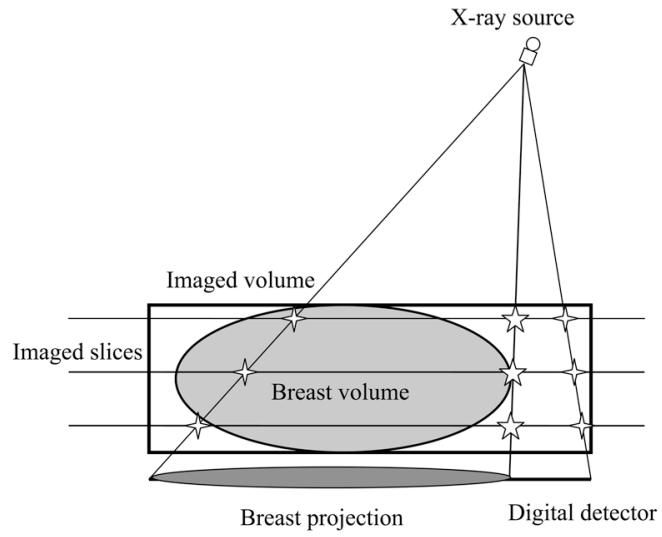

(b)

FIG. 5. Breast boundary artifacts (indicated by open stars) and detector boundary artifacts (indicated by open crosses) appear on all tomosythesized slices along the x-ray path for one x-ray source position. The imaged volume is defined as the volume enclosed by the rectangular box while the breast volume is that enclosed by the ellipse. This example shows the situations (a) that the breast boundary is completely contained within the detector boundary; (b) that one side of the breast boundary is cut off by the detector boundary due to both the large breast size and the wide projection angle.

angles, in the cases of very large breast sizes, or at the top part of the pectoral muscle on most MLO views.

As an example, Fig. 6(a) shows a patient CC-view DTM slice reconstructed by the SART method with one iteration. The breast boundary artifacts were clearly seen as repeated breast-shaped shadows and the detector boundary artifacts as multiple horizontal lines in both the top and the bottom areas.

The breast boundary artifacts generally will be enhanced, rather than reduced, by the SART method as the number of iteration increases until a convergent solution is reached. This is because the SART method, similar to other iterative reconstruction algorithms, acts like an unsharp-masking (bandpass) filter in updating the imaged volume and thus favors edge enhancement. The convergent solution, however, will generally be very noisy due to the severe ill-posed nature of the inverse problem.

For detector boundary artifacts, they may be enhanced or reduced in the process of approaching the convergent solu- 


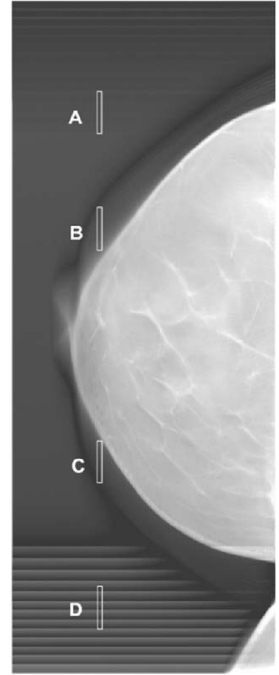

(a)

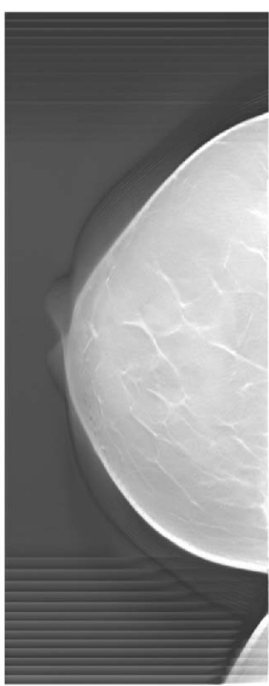

(b)

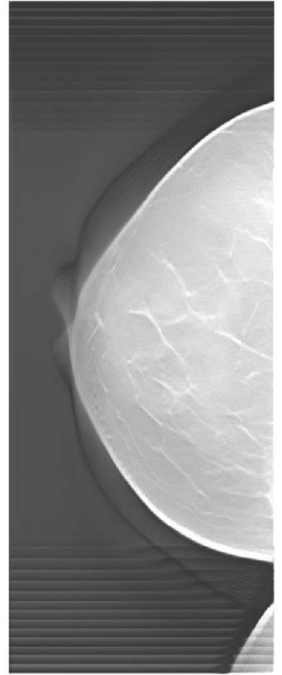

(c)
FIG. 6. One selected tomosynthesized slice obtained by SART reconstruction method with (a) one, (b) two, and (c) three iterations, without using any breast boundary information. The breast boundary artifacts were clearly observed as repeated breast-shaped shadows while the detector boundary artifacts as multiple horizontal lines in both the top and the bottom areas. The same window level and window width were used for display of the images. The artifacts did not diminish as the number of iterations increased. Open rectangles in (a) indicate the regions of interest for which the line profiles are compared in Fig. 7.

tion, depending on the access order of the PVs in the iterative reconstruction process. To demonstrate these effects, we applied the SART reconstruction method to the patient CCview DTM up to three iterations, and presented the result of the same tomosynthesized slice in Figs. 6(b) and 6(c), respectively. A constant relaxation parameter of 0.5 was used for all three SART iterations. Four regions of interest, depicted by open rectangles in Fig. 6(a), were selected for comparison. Regions A and D are located in the detector boundary artifact area while regions $\mathrm{B}$ and $\mathrm{C}$ in the breast boundary artifact area. The line profiles in these four regions are shown in Fig. 7. The enhancement of both types of boundary artifacts by SART iterations can be observed clearly in regions $\mathrm{A}, \mathrm{B}$ and $\mathrm{C}$. In region $\mathrm{D}$, the detector boundary artifacts were more prominent than those in region A at the upper part. This difference can be attributed to the access order of the PV images employed in SART reconstruction of this example, sequentially from that with the $\mathrm{x}$-ray source at the top side to the bottom side. ${ }^{45}$ The upper part of the imaged volume were covered completely and thus updated uniformly by the first eight PVs at the beginning of the iteration. The voxels of the entire region therefore attained reasonable values before the subsequent PVs that partially covered the upper part of the imaged volume started to generate the detector boundary artifacts. On the contrary, the artifacts at the lower part were generated starting from the first PV. The large difference between the initialization constant values outside the PV image and the updated voxel values within the PV image resulted in large discontinuity in the voxel values across the detector boundary. Although the
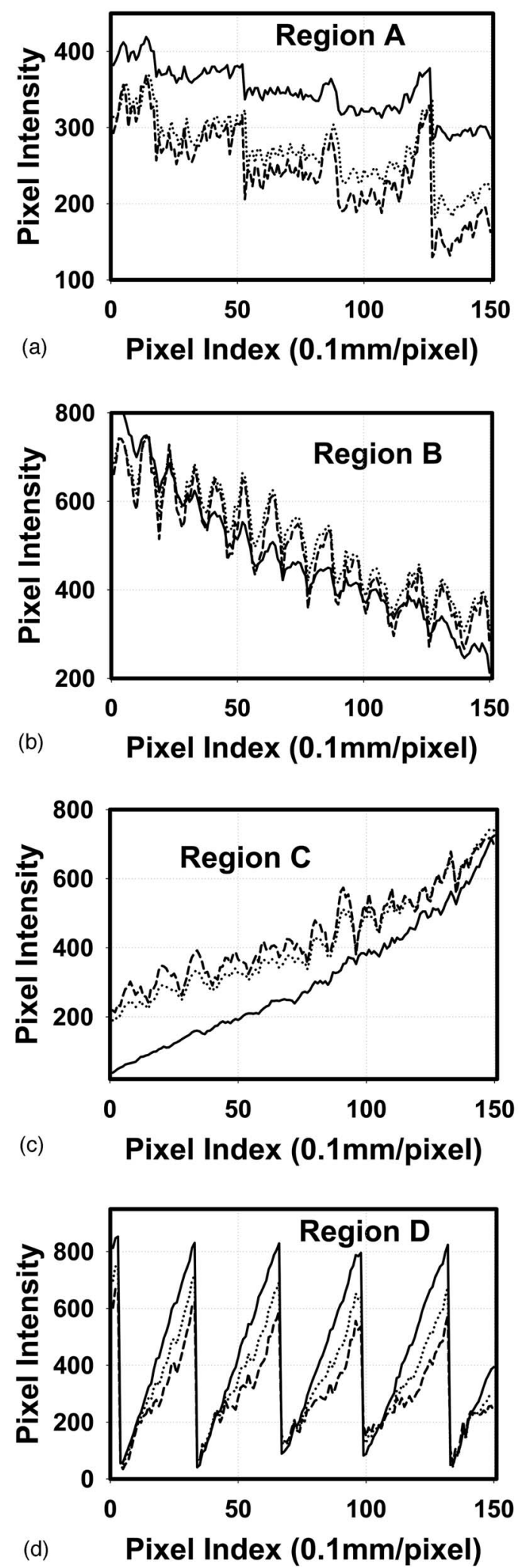

FIG. 7. comparison of line profiles extracted from selected detector boundary artifact areas (regions A and D) and breast boundary artifact areas (regions B and C) of SART results with one (solid line), two (dotted line) and three iterations (dashed line). The locations of these selected regions are indicated by open rectangle in Fig. 6(a). Pixel index always starts from the end that is close to the bottom part of the tomosynthesized slice. 
subsequent iterations still enhanced the edges, their dominant effect was to bring the average voxel values to the level of those of air attenuation. The overall result was the apparent reduction of edge artifacts with further iterations in region $\mathrm{D}$, opposite to those observed in the other regions.

To verify these, we continued the SART reconstruction up to ten iterations and observed that the iteration appeared to have reached a convergent solution for this DTM case (results not shown here). The reconstructed background was close to the local background level and the boundary artifacts became more symmetric in the upper and lower parts of the slices. The line profiles of regions A and D in the convergent result exhibited similar detector boundary artifacts which did not vanish with further iterations. Note that, because the large number of iterations causes over enhancement of the breast structures and excessive computation time, the reconstruction will generally terminate long before it reaches the convergent solution. We have found in our previous study ${ }^{25}$ that good-quality patient DTMs can be achieved after only one iteration with SART.

\section{II.D. Application of the 2D and 3D breast boundary information to SART reconstruction}

We evaluated the application of breast boundary information to DTM reconstruction in two ways with the SART method. First, when we update the imaged volume in the iterative process, the re-projection and the back-projection of the difference between the calculated data and the measured data will only be performed along those "valid" rays within the $2 \mathrm{D}$ breast area. We will not use any other rays and thus the voxels in the imaged volume associated with these "invalid" rays are not updated and stay at the current values. Second, after one complete SART iteration has been performed, we use the 3D breast surface to mask the resulting volume and set the values of all voxels outside the breast surface to that of air.

In terms of Eq. (4), the use of the 2D breast curves and the 3D breast surface can each be expressed as a linear operator in matrix form, denoted by diagonal matrices $\mathbf{P}_{n}$ $\in R^{I \times I}$ and $\mathbf{Q} \in R^{J \times J}$, respectively, where the diagonal element of $\mathbf{P}_{n}$ is 1 if the corresponding detector element is marked as breast area in the $n$th PV, and 0 as air area; similarly, the diagonal element of $\mathbf{Q}$ is 1 if the corresponding voxel is marked as breast volume, and 0 as air volume. By using these linear operators, the SART method can be modified as follows:

$$
\begin{aligned}
& \mathbf{x}^{n}=\mathbf{x}^{n-1}+\lambda \cdot \mathbf{M}_{n} \mathbf{A}_{n}^{T} \mathbf{W}_{n} \mathbf{P}_{n}\left(\mathbf{y}_{n}-\mathbf{A}_{n} \mathbf{x}^{n-1}\right) \\
& \mathbf{x}^{N}=\mathbf{Q} \mathbf{x}^{N} .
\end{aligned}
$$

Note the operator $\mathbf{Q}$ is applied after one SART iteration is completed.

Our proposed method reduces the computational effort by eliminating the processing of all rays outside the breast region on each individual PV. Thus the improved efficiency ratio can be defined as the summation of the background

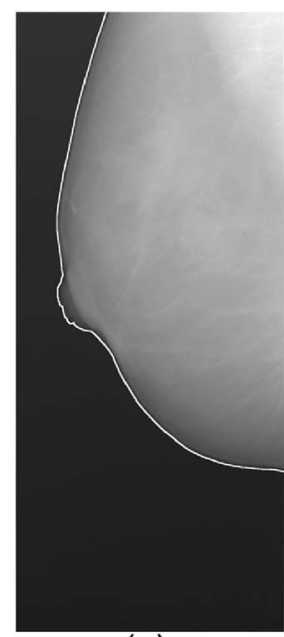

(a)

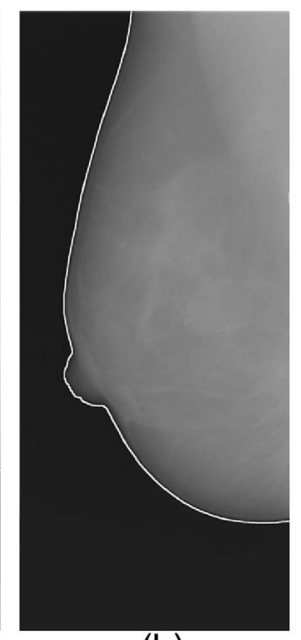

(b)

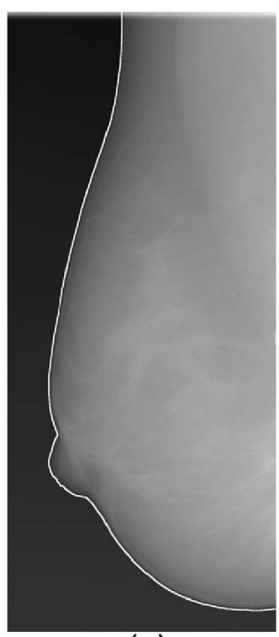

(c)
FIG. 8. Projection view images of a DTM in MLO view: (a) PV at $-30^{\circ}$, (b) $\mathrm{PV}$ at $0^{\circ}$, and (c) $\mathrm{PV}$ at $+30^{\circ}$. The $\mathrm{x}$-ray source moved in the vertical direction relative to the images shown. The detected breast boundary was indicated by the white solid line on each PV image

areas outside the detected breast boundary on all PV images divided by the product of the detector area and the number of PVs.

\section{RESULTS}

The proposed method for artifact reduction has been evaluated by using patient PV images acquired with the second generation GE prototype DTM system. Institutional Review Board approval and informed consent were obtained for collection of the patient cases. DTM images were acquired in two views: a cranio-caudal (CC) view and a mediolateral oblique (MLO) view. In the rest of the paper, only image results of MLO view were shown without loss of generality. CC view results were similar to those of MLO views on the side without the pectoral muscle. The No. 1, No. 11 and No. 21 raw PV images of MLO view, corresponding to the projection angles $-30^{\circ}, 0^{\circ},+30^{\circ}$, are shown in Fig. 8 . The detected breast boundaries were indicated by solid white lines. The x-ray source moved in the vertical direction relative to the images shown. Figure 9 shows the generated 3D

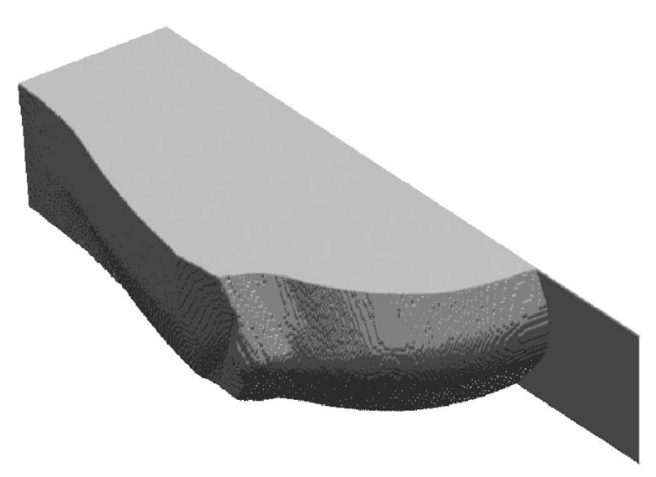

FIG. 9. The 3D breast surface generated from 2D breast boundary curves for MLO view by using the $3 \mathrm{D}$ conical trimming method. 


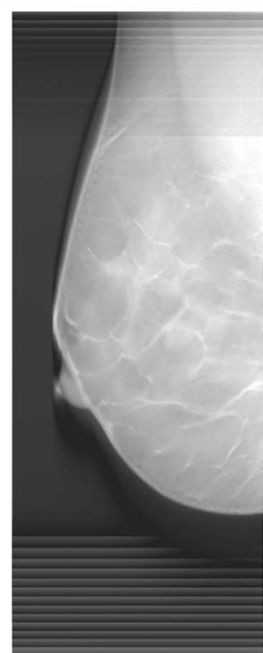

(a)

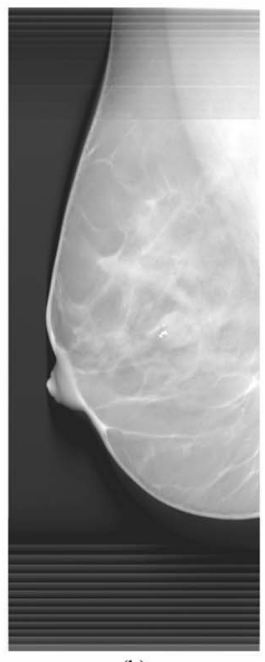

(b)

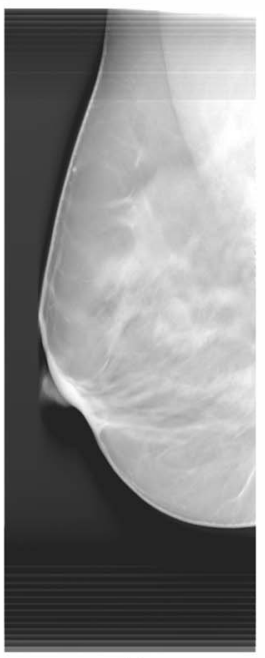

(c)

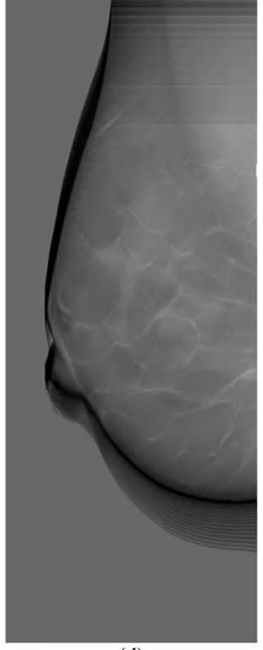

(d)

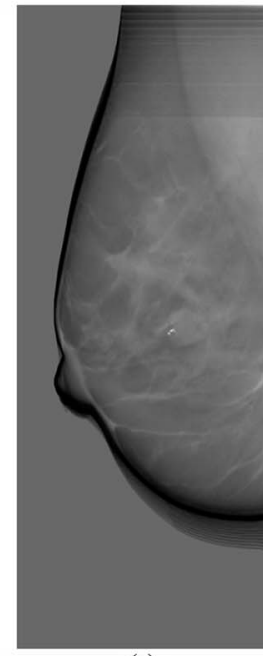

(e)

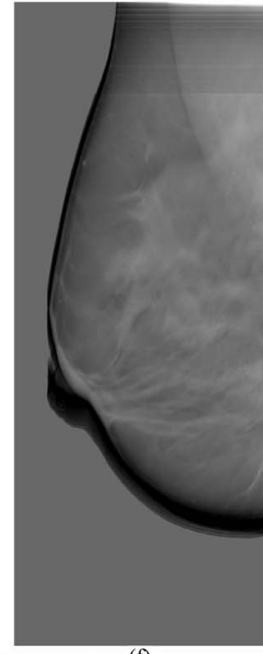

(f)

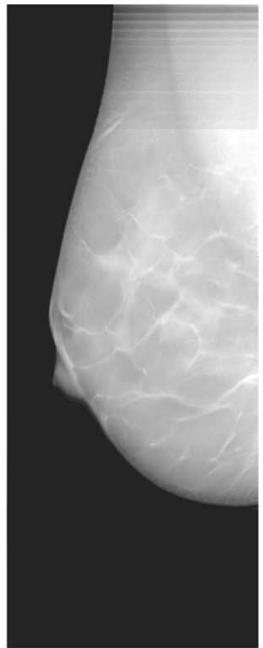

(g)

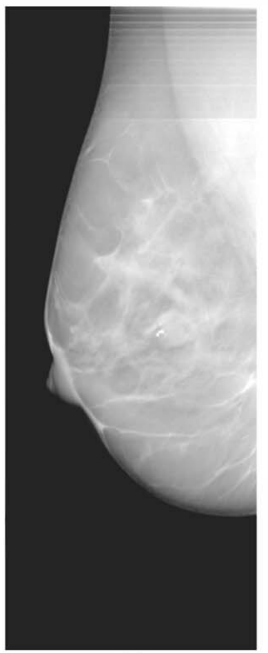

(h)

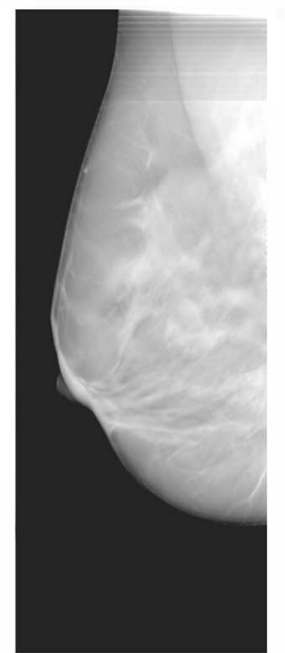

(i)

FIG. 10. Three selected tomosynthesized slice obtained by SART reconstruction for MLO view: (a), (d), and (g) $3.9 \mathrm{~cm}$, (b), (e) and (h) $3.3 \mathrm{~cm}$, (c), (f), and (i) $2.3 \mathrm{~cm}$ above the breast support plate; (a)-(c) reconstructed slices without using any breast boundary information, (d)-(f) reconstructed slices with using only 2D breast mask information (g)-(i) reconstructed slices with both the 2D and 3D breast boundary information. Note that with 2D breast mask, the detector boundary artifacts at the bottom have been eliminated while the breast boundary artifacts have been totally removed after application of the $3 \mathrm{D}$ breast shape. The skin lines are also less over-enhanced. The detector boundary artifacts at the top of the slice do not change because the tissue extended beyond the detector boundary. SART results with different methods ((a)-(c), (d)-(f), and (g)-(i)) are displayed with different window width and level to achieve visually comparable contrast of the boundary artifacts.

breast surface from the MLO view by using the proposed 3D conical trimming method.

Figure 10 shows examples of MLO-view DTM slices reconstructed with the SART method using one iteration and a relaxation parameter of 0.5 . A constant distribution of voxel values was used as initialization. The DTM images reconstructed without using boundary information, as shown in (a)-(c); with only 2D breast mask information, as shown in (d)-(f); and with both 2D mask and 3D breast shape information, as shown in (g)-(i), were compared. The results of SART method with only 3D breast shape information should be the same as those with both 2D and 3D breast information, but one will not gain computational efficiency. The three chosen slices are $3.9,3.3$, and $2.3 \mathrm{~cm}$ above the breast support plate and the thickness of the imaged breast was measured by the DTM system as $5.4 \mathrm{~cm}$. While the choice of these tomosynthesized slices was somewhat arbitrary, we attempt to choose slices that can clearly demonstrate the difference between the results with and without using breast boundary information. Note that in Figs. 10(d)-10(f), the background voxels retained the initialization constant as their values, which was chosen to be the average linear attenuation coefficient of normal breast tissues and was higher than those of the fatty tissue. In contrast, the exterior region outside the breast in Figs. 10(g)-10(i) were set to have values of zero, which was approximately the attenuation coefficient of air, after application of the 3D breast shape information.

Without using any breast boundary information, the detector boundary artifacts were clearly observed as multiple horizontal lines especially at the bottom of all three tomosynthe- 
sized slices. At the top part of these slices, there were also detector boundary artifacts, as indicated by the PV-count map shown in Fig. 4, but the artifacts were much weaker than those at the bottom as a result of the PV access order, discussed above. The breast boundary artifacts, represented as repeated breast-shaped ghosts around the boundary, were clearly observed around the true breast boundary in-focus on each slice. The number of ghost shadows is 20 if the breast boundary is imaged in all $21 \mathrm{PVs}$ because one boundary is in-focus and appears as real breast boundary at that depth.

The application of the generated 3D breast surface to the reconstructed slices removed all breast boundary artifacts. With the 2D breast mask information incorporated, the detector boundary artifacts outside the breast have been eliminated. More importantly, the skin lines in all DTM images are less over enhanced when the reconstruction is limited to the valid rays within the $2 \mathrm{D}$ breast boundary. The overenhanced skin lines may create skin-thickening artifacts. The remaining detector artifacts within the generated breast surface, as shown at the top part of the slices, are due to the fact that the pectoral muscle was projected beyond the detector boundary at the large tomosynthesis angles, as shown in Fig. 8.

In this case, the computation time was reduced by $76.3 \%$ and $69.9 \%$ for the CC and MLO views, respectively, by using the $2 \mathrm{D}$ breast boundary masks for all PVs.

\section{DISCUSSION}

We have investigated the application of the 2D and 3D breast boundary information to DTM reconstruction with the SART method. Preliminary results show that this method can substantially reduce the computation time and effectively remove the breast and detector boundary artifacts on tomosynthesized slices by employing only projection information from the rays intersecting the breast volume for reconstruction. When the breast boundary is completely inside the detector boundary, the use of valid rays only within the breast areas for reconstruction excludes the transition regions in the imaged volume that are covered by different number of PVs and enhanced during updating in subsequent PV processing. The detector boundary artifacts can thus be totally removed. When the projected breast image exceeds the detector area, the detector boundary artifacts outside the breast volume can be removed but those inside remain where the breast PV images are cut off by the detector boundary. After the SART reconstruction, breast boundary artifacts were removed by trimming off the air volume outside the 3D breast surface. The investigation of methods for reduction of the remaining artifacts will be undertaken in the future.

The 2D and 3D breast boundary information can be obtained prior to the reconstruction process. The $2 \mathrm{D}$ breast boundary curves are extracted from the individual PV images with a segmentation method developed in our laboratory. The $3 \mathrm{D}$ breast surface is generated by back projecting the $2 \mathrm{D}$ breast boundary curves sequentially followed by a $3 \mathrm{D}$ conical trimming process. The computational effort involved is trivial compared to the reconstruction procedure because only boundary pixels are needed to be back projected. Note that the 3D conical trimming method cannot recover parts of the breast surface where they overlap projected breast regions in all PV images. This will occur most likely in the region close to the gap between the breast support plate and the lower part, and between the compression plate and the upper part, around the boundary of the compressed breast, as indicated by dashed arrows in Fig. 3. The size of the breast surface area that cannot be recovered due to missing information will depend on the angular range of the DTM system, and the thickness and boundary curvature of the compressed breast. The larger the angular range of the DTM system, the smaller the missing gap.

The efficiency ratio is defined by dividing the average background area outside the detected breast boundary over all PV images by the detector area. Therefore the saving on the computational effort depends on the breast size of individual patient case and is greater for smaller breasts. To estimate the average saving on computational effort in a random sample of patient cases, we have calculated the efficiency ratio as the background area to detector area ratio for a data set of 96 two-view full field digital mammograms (FFDMs) acquired with a GE Senographe 2000D system, the detector of which has the same dimension as that of our prototype DTM system. This data set was collected previously in the Department of Radiology at the University of Michigan for our CAD study. ${ }^{46}$ The resulting mean efficiency ratio for $96 \mathrm{CC}$-view FFDMs is $65.2 \%$ with a standard deviation of $14.7 \%$; and the mean value for 96 MLOview FFDMs is $56.0 \%$ with a standard deviation of $13.5 \%$. While the average background area in the 21 PVs of a DTM will be slightly different from that of an FFDM, this should provide a good estimate of the efficiency ratio.

The developed 2D and 3D breast boundary information can be used in different ways with DTM reconstruction algorithms, rather than the one we described in this work. For example, with SART or other iterative methods, the 3D breast surface can be employed at different stages in the iterative process, e.g., masking the resulting imaged volume to allow setting all voxels outside the breast surface to air after each PV image has been used, rather than after all PV images have been used exactly once, as we suggested in this work. In terms of using object shape information in image reconstruction, Tam has presented a simple method to construct the convex hull of an imaged object in 2D limitedangle computerized tomography (CT) by intersecting or superimposing the back-projected object shadows. ${ }^{47} \mathrm{He}$ utilized this object support information in an iterative limited-angle CT reconstruction in which the missing PV images are estimated and used in a full-range FBP method. During the iterative process, the reconstructed object density distribution is improved by correcting the external volume to zero and applying other constraints to the density range. ${ }^{48}$

Another potential application of the 3D breast surface information is quantitative DTM reconstruction. In DTM reconstruction, the air volume outside the breast, if not excluded, will affect the estimation of the average attenuation value back projected to the real breast volume. With the $3 \mathrm{D}$ 
breast surface information, if only the pathlength within the real breast volume will be taken into account in calculating the $\mathrm{x}$-ray attenuation properties of the breast tissues, it will provide potential improvement in quantitative estimation of the linear attenuation coefficients.

In addition to applications in DTM reconstruction, the 3D breast surface and volume information will be useful for breast density estimation. Breast density has been shown to be an important risk factor for breast cancer. The change in breast density is considered a useful surrogate for estimating the change in breast cancer risk due to treatment or intervention. Currently the change in breast density is monitored mainly by estimating the change in the dense area on conventional projection mammograms. Because of the projection of the 3D volume to a $2 \mathrm{D}$ plane, the percentage of dense area on a mammogram does not accurately reflect the percentage of dense tissue in the breast volume although a strong correlation has been demonstrated. ${ }^{49}$ It can be expected that 3D volumetric information available from DTM will provide a better estimation of the amount of dense tissue in the breast volume and its changes over time than projection mammograms.

\section{CONCLUSION}

In this study, we have applied the 2D breast boundary information and the generated 3D breast surface to SART reconstruction in breast tomosynthesis mammography. The 2D breast boundary is detected on the projection view images using a boundary tracking algorithm developed in our laboratory and the 3D breast surface is generated with a $3 \mathrm{D}$ conical trimming method. The $2 \mathrm{D}$ breast boundary curves on PV images are used to restrict the SART reconstruction to be performed only inside the breast area while the 3D breast surface is used to exclude reconstruction artifacts outside the breast volume. Experimental results with patient PV images demonstrated that the proposed method can substantially improve computational efficiency by eliminating unnecessary reconstruction in regions outside the breast. Both breast boundary and detector boundary artifacts can be effectively removed by the proposed method.

\section{ACKNOWLEDGMENTS}

This work is supported by USPHS Grant Nos. CA120234, CA91713 and CA95153, and U.S. Army Medical Research and Materiel Command Grant Nos. W81XWH-07-1-0324 and DAMD 17-02-1-0214. The content of this paper does not necessarily reflect the position of the funding agencies and no official endorsement of any equipment and product of any companies mentioned should be inferred. The digital tomosynthesis system was developed by the GE Global Research Group through the Biomedical Research Partnership (USPHS Grant No. CA91713) collaboration.

\footnotetext{
${ }^{a)}$ Electronic mail: yihzhang@med.umich.edu

${ }^{1}$ L. T. Niklason et al., "Digital tomosynthesis in breast imaging," Radiology 205, 399-406 (1997).

${ }^{2} \mathrm{~T}$. Wu et al., "Tomographic mammography using a limited number of low-dose cone-beam projection images," Med. Phys. 30, 365-380 (2003).
}

${ }^{3}$ S. Suryanarayanan, A. Karellas, S. Vedantham, S. J. Glick, C. J. D’Orsi, S. P. Baker, and R. L. Webber, "Comparison of tomosynthesis methods used with digital mammography," Acad. Radiol. 7, 1085-1097 (2000).

${ }^{4}$ S. Suryanarayanan, A. Karellas, S. Vedantham, S. P. Baker, S. J. Glick, C. J. D'Orsi, and R. L. Webber, "Evaluation of linear and nonlinear tomosynthetic reconstruction methods in digital mammography," Acad. Radiol. 8, 219-224 (2001).

${ }^{5}$ J. W. Eberhard et al., "High speed, large angle mammography tomosynthesis system," Proc. SPIE 6142, 61420C (2006).

${ }^{6}$ D. Kopans, "Digital tomosynthesis and other applications," RSNA Program Book 2005130 (2005).

${ }^{7}$ E. Rafferty, "Breast tomosynthesis," RSNA Program Book 2005141 (2005).

${ }^{8}$ M. A. Helvie, M. A. Roubidoux, Y. Zhang, P. L. Carson, and H.-P. Chan, "Tomosynthesis mammography vs conventional mammography: Lesion detection and reader preference. Initial experience," RSNA Program Book 2006335 (2006)

${ }^{9}$ B. Ren, C. Ruth, J. Stein, A. Smith, I. Shaw, and Z. Jing, "Design and performance of the prototype full field breast tomosynthesis system with selenium based flat panel detector," Proc. SPIE 5745, 550-561 (2005).

${ }^{10} \mathrm{M}$. Bissonnette et al., "Digital breast tomosynthesis using an amorphous selenium flat panel detector," Proc. SPIE 5745, 529-540 (2005).

${ }^{11}$ S. Poplack, C. Kogel, and H. Nagy, "Initial experience with digital breast tomosynthesis in 99 breasts of 98 women with abnormal digital screening mammography," RSNA Program Book 2005317 (2005).

${ }^{12}$ R. Moore, A. Stewart, T. Wu, D. Kopans, E. Rafferty, and D. GeorgianSmith, "Second-generation digital breast tomosynthesis (DBT) in the screening setting: Workflow and preliminary results," RSNA Program Book 2005318 (2005).

${ }^{13}$ J. Lo, J. Baker, J. Orman, T. Mertelmeier, and S. Singh, "Breast tomosynthesis: Initial clinical experience with 100 human subjects," RSNA Program Book 2006335 (2006).

${ }^{14}$ D. G. Grant, "Tomosynthesis: A three-dimensional radiographic imaging technique," IEEE Trans. Biomed. Eng. 19, 20-28 (1972).

${ }^{15}$ Z. Kolitsi, G. Panayiotakis, V. Anastassopoulus, A. Scodras, and N. Pallikarakis, "A multiple projection method for digital tomosynthesis," Med. Phys. 19, 1045-1050 (1992).

${ }^{16}$ A. Kak and M. Slaney, Principle of Computerized Tomographic Imaging (IEEE, New York, 1988).

${ }^{17} \mathrm{G}$. Lauritsch and W. Haerer, "A theoretical framework for filtered backprojection in tomosynthesis," Proc. SPIE 3338, 1127-1137 (1998).

${ }^{18}$ H. Matsuo, A. Iwata, I. Horiba, and N. Suzumura, "Three-dimensional image reconstruction by digital tomosynthesis using inverse filtering," IEEE Trans. Med. Imaging 12, 307-313 (1993).

${ }^{19}$ T. Mertelmeier, J. Orman, W. Haerer, and M. K. Dudam, "Optimizing filtered backprojection reconstruction for a breast tomosynthesis prototype device," Proc. SPIE 6142, 61420F (2006).

${ }^{20}$ J. T. Dobbins III and D. J. Godfrey, "Digital x-ray tomosynthesis: Current state of the art and clinical potential," Phys. Med. Biol. 48, R65-R106 (2003).

${ }^{21}$ R. J. Warp, D. J. Godfrey, and J. T. Dobbins III, “Applications of matrix inverse tomosynthesis," Proc. SPIE 3977, 376-383 (2000).

${ }^{22}$ Y. Chen, J. Y. Lo, J. A. Baker, and J. T. Dobbins III, "Gaussian frequency blending algorithm with matrix inversion tomosynthesis (MITS) and filtered back projection (FBP) for better digital breast tomosynthesis reconstruction," Proc. SPIE 6142, 122-130 (2006).

${ }^{23}$ B. Wang, K. Barnera, and D. Leeb, "Algebraic tomosynthesis reconstruction,” Proc. SPIE 5370, 711-718 (2004).

${ }^{24}$ A. H. Andersen and A. C. Kak, "Simultaneous algebraic reconstruction technique (SART): A new implementation of the ART algorithm," Ultrason. Imaging 6, 81-94 (1984).

${ }^{25}$ Y. Zhang, H.-P. Chan, B. Sahiner, J. Wei, M. M. Goodsitt, L. M. Hadjiiski, J. Ge, and C. Zhou, "A comparative study of limited-angle conebeam reconstruction methods for breast tomosynthesis," Med. Phys. 33, 3781-3795 (2006).

${ }^{26} \mathrm{~K}$. Lange and J. A. Fessler, "Globally convergent algorithms for maximum a posteriori transmission tomography," IEEE Trans. Image Process. 4, 1430-1438 (1995).

${ }^{27}$ T. Wu, J. Zhang, R. Moore, E. Rafferty, and D. Kopans, "Digital tomosynthesis mammography using a parallel maximum-likelihood reconstruction method," Proc. SPIE 5368, 1-11 (2004).

${ }^{28}$ U. E. Ruttimann, R. A. J. Groenhuis, and R. L. Webber, "Restoration of digital multiplane tomosynthesis by a constrained iteration method," 
IEEE Trans. Med. Imaging MI-3, 141-148 (1984)

${ }^{29}$ D. N. G. Roy, R. A. Kruger, B. Yih, and P. D. Rio, "Selective plane removal in limited angle tomographic imaging," Med. Phys. 12, 65-70 (1985).

${ }^{30}$ Z. Kolitsi, G. Panayiotakis, and N. Pallikarakis, "A method for selective removal of out-of-plane structures in digital tomosynthesis," Med. Phys. 20, 47-50 (1993).

${ }^{31}$ J. Ge, H.-P. Chan, B. Sahiner, Y. Zhang, J. Wei, L. M. Hadjiiski, and C. Zhou, "Digital tomosynthesis mammography: Intra- and interplane artifact reduction for high-contrast objects on reconstructed slices using a priori 3D geometrical information," Proc. SPIE 6512, 65124Q (2007).

${ }^{32}$ P. Haaker, E. Klotz, R. Koppe, R. Linde, and H. Moller, "A new digital tomosynthesis method with less artifacts for angiography," Med. Phys. 12, 431-436 (1985)

${ }^{33}$ B. E. H. Claus and J. W. Eberhard, "A new method for 3D reconstruction in digital tomosynthesis,” Proc. SPIE 4684, 814-824 (2002).

${ }^{34}$ T. Wu, R. H. Moore, and D. B. Kopans, "Voting strategy for artifact reduction in digital breast tomosynthesis," Med. Phys. 33, 2461-2471 (2006).

${ }^{35}$ I. Goddard, T. Wu, S. Thieret, A. Berman, and H. Bartsch, "Implementing an iterative reconstruction algorithm for digital breast tomosynthesis on graphics processing hardware,” Proc. SPIE 6142, 61424V (2006).

${ }^{36}$ H.-P. Chan, J. Wei, T. Wu, B. Sahiner, E. A. Rafferty, L. M. Hadjiiski, M. A. Helvie, M. A. Roubidoux, R. H. Moore, and D. B. Kopans, "Computer-aided detection on digital breast tomosynthesis (DBT) mammograms - dependence on image quality of reconstruction," RSNA Program Book 269 (2005).

${ }^{37}$ R. L. Siddon, "Fast calculation of the exact radiological path for a threedimensional CT array,” Med. Phys. 12, 252-255 (1985).

${ }^{38}$ T. Wu, R. H. Moore, E. A. Rafferty, and D. B. Kopans, "A comparison of reconstruction algorithms for breast tomosynthesis," Med. Phys. 31, 2636-2647 (2004).

${ }^{39}$ C. Zhou, H.-P. Chan, C. Paramagul, M. A. Roubidoux, B. Sahiner, L. M.
Hadjiiski, and N. Petrick, "Computerized nipple identification for multiple image analysis in computer-aided diagnosis," Med. Phys. 31, 28712882 (2004).

${ }^{40}$ Y.-T. Wu, C. Zhou, L. M. Hadjiiski, J. Shi, C. Paramagul, B. Sahiner, and H.-P. Chan, "A dynamic multiple thresholding method for automated breast boundary detection in digitized mammograms," Proc. SPIE 6512, 65122U (2007)

${ }^{41}$ N. Otsu, "A threshold selection method from gray-level histograms," IEEE Trans. Syst. Man Cybern. 9, 62-66 (1979).

${ }^{42}$ A. H. Andersen, "Algebraic reconstruction in CT from limited views," IEEE Trans. Med. Imaging 8, 50-55 (1989).

${ }^{43}$ R. Gordon, R. Bender, and G. T. Herman, "Algebraic reconstruction techniques (ART) for three dimensional electron microscopy and $\mathrm{X}$-ray photography," J. Theor. Biol. 29, 471-481 (1970).

${ }^{44} \mathrm{G}$. T. Herman, Image Reconstruction from Projections: The Fundamentals of Computerized Tomography (Academic, New York, 1980).

${ }^{45}$ Y. Zhang, H.-P. Chan, B. Sahiner, J. Wei, L. M. Hadjiiski, J. Ge, and C. Zhou, "Breast tomosynthesis reconstruction with simultaneous algebraic reconstruction technique (SART): Effect of access strategy of projectionview images," RSNA Program Book 130 (2006).

${ }^{46}$ J. Ge, B. Sahiner, L. M. Hadjiiski, H.-P. Chan, J. Wei, M. A. Helvie, and C. Zhou, "Computer aided detection of clusters of microcalcifications on full field digital mammograms," Med. Phys. 33, 2975-2988 (2006).

${ }^{47} \mathrm{~K}$. C. Tam, "The construction and use of convex hulls in limited-angle computerized tomography," J. Nondestruct. Eval. 6, 189-204 (1987).

${ }^{48}$ K. C. Tam and V. Perez-Mendez, "Tomographical imaging with limitedangle input," J. Opt. Soc. Am. 71, 582-592 (1981).

${ }^{49}$ J. Wei, H.-P. Chan, M. A. Helvie, M. A. Roubidoux, B. Sahiner, L. Hadjiiski, C. Zhou, S. Paquerault, T. Chenevert, and M. M. Goodsitt, "Correlation between mammographic density and volumetric fibroglandular tissue estimated on breast MR images," Med. Phys. 31, 933-942 (2004). 